\title{
BERNSTEIN-SATO POLYNOMIALS ON NORMAL TORIC VARIETIES
}

\author{
JEN-CHIEH HSIAO AND LAURA FELICIA MATUSEVICH
}

\begin{abstract}
We generalize the Bernstein-Sato polynomials of Budur, Mustaţă, and Saito to ideals in normal semigroup rings. In the case of monomial ideals, we also relate the roots of the BernsteinSato polynomial to the jumping coefficients of the corresponding multiplier ideals. In order to prove the latter result, we obtain a new combinatorial description for the multiplier ideals of a monomial ideal in a normal semigroup ring.
\end{abstract}

\section{INTRODUCTION}

Let $f \in \mathbb{C}[x]=\mathbb{C}\left[x_{1}, \ldots, x_{n}\right]$ be a non-constant polynomial and let $D=\mathbb{C}\left[x, \partial_{x}\right]$ be the Weyl algebra. The Bernstein-Sato polynomial (or $b$-function) of $f$, introduced independently in [Ber72] and [SS72], is the monic polynomial $b_{f}(s) \in \mathbb{C}[s]$ of smallest degree such that there exists $P(s) \in$ $D[s]=D \otimes_{\mathbb{C}} \mathbb{C}[s]$ satisfying the functional equation $P(s) \cdot f^{s+1}=b_{f}(s) f^{s}$. It is well known that $b_{f}(s)=s+1$ if and only if the hypersurface defined by $f$ is nonsingular. Moreover, the roots of $b_{f}(s)$ are related to the eigenvalues of the Milnor monodromy of $f$ [Mal83], and the poles of the local zeta function associated to $f$ [Igu00].

Another important singularity invariant are the multiplier ideals $\mathcal{J}\left(\mathbb{C}^{n}, \alpha\langle f\rangle\right)$ associated to the hypersurface $f$ in $\mathbb{C}^{n}$ [Laz04]. They can be defined via an embedded log resolution of the pair $\left(\mathbb{C}^{n},\langle f\rangle\right)$. When the coefficient $\alpha$ varies, the multiplier ideal $\mathcal{J}\left(\mathbb{C}^{n}, \alpha\langle f\rangle\right)$ jumps. In fact, the smallest jumping coefficient of $\left(\mathbb{C}^{n},\langle f\rangle\right)$ (i.e. the log-canonical threshold of the hypersurface defined by $f$ ) is the smallest root of $b_{f}(-s)$ [Yan83, Lic89, Kol97]. Generalizing this result, one of the main theorems in [ELSV04] states that if $\xi$ is a jumping coefficient in $(0,1]$ of the pair $\left(\mathbb{C}^{n},\langle f\rangle\right)$, then it is a root of $b_{f}(-s)$. Along the same lines, Budur, Mustaţă, and Saito extended the notion of $b$-functions to the case of arbitrary ideals on smooth affine varieties [BMS06a]. Utilizing the theory of $V$-filtrations from [Kas83] and [Mal83], they showed that the $b$-function of an ideal is independent of the choice of generators. Furthermore, they generalized the connection established in [ELSV04] between the roots of their $b$-functions and the jumping coefficients of multiplier ideals to this more general setting.

Theorem 1.1 ([BBS06a $]$ ). Let I be an ideal on a smooth affine variety $X$ over $\mathbb{C}$. Then the logcanonical threshold of the pair $(X, I)$ coincides with the smallest root $\alpha_{I}$ of the b-function $b_{I}(-s)$, and any jumping coefficient of $(X, I)$ in $\left[\alpha_{I}, \alpha_{I}+1\right)$ is a root of $b_{I}(-s)$.

As the theory of multiplier ideals is generalized to the case of arbitrary ideals on normal varieties [dFH09], it is interesting to explore possible generalizations of Theorem 1.1] To this end, the first task is to seek for a suitable candidate that plays the role of the Weyl algebra. In the current work, we restrict to the case where the ambient variety $X$ is an affine normal toric variety. There

2010 Mathematics Subject Classification. Primary: 14F10; Secondary: 14M25, 14F18, 14B05.

JCH was partially supported by MOST grant 105-2115-M-006-015-MY2.

LFM was partially supported by NSF grant DMS 1500832 . 
exists an explicit combinatorial description of Grothendieck's ring of differential operators $D_{X}$ on the toric variety $X$ [Mus87, Jon94, ST01], serving as an analog of Weyl algebra in the case of $X=\mathbb{C}^{n}$. We apply this description of $D_{X}$ and the results in [BMS06a, BMS06b] to construct the $b$-function $b_{I}(s)$ for an ideal $I$ on the toric variety $X$. To state our results more explicitly, let us set up some notations that will be used throughout this paper.

Notation 1.2. 1. Throughout this article, we work over an algebraically closed field $\mathbb{k}$ of characteristic 0 instead of the complex number field $\mathbb{C}$. Our results are valid in this setting. The coordinate ring of the affine normal toric variety $X$ is represented by the semigroup ring $\mathbb{k}[\mathbb{N} A]$ generated by the columns $a_{1}, \ldots, a_{d}$ of a rank $d$ matrix $A \in \mathbb{Z}^{d \times m}$. We assume that $\mathbb{Z} A=\mathbb{Z}^{d}$, the cone $C=\mathbb{R}_{>0} A$ in $\mathbb{R}^{d}$ over $A$ is strongly convex, and the semigroup $\mathbb{N} A$ is normal, meaning that $C \cap \mathbb{Z}^{d}=\mathbb{N} A$. The faces of $C$ are denoted by greek letters $\sigma, \tau$ etc. This may refer to an index set, to a collection of columns of $A$, or to the actual face of the cone. If $\sigma$ is a facet of $C$, define its primitive integral support function $F_{\sigma}: \mathbb{R}^{d} \rightarrow \mathbb{R}$ such that
(a) $F_{\sigma}(\mathbb{Z} A)=\mathbb{Z}$
(b) $F_{\sigma}\left(a_{i}\right) \geq 0$ for $i=1, \ldots, n$,
(c) $F_{\sigma}\left(a_{i}\right)=0$ for $a_{i} \in \sigma$.

Define the linear map $F: \mathbb{R}^{d} \rightarrow \mathbb{R}^{\mathscr{F}}$ by $F(p)=\left(F_{\sigma}(p)\right)_{\sigma \in \mathscr{F}}$, where $\mathscr{F}$ is the collection of facets of $C$.

2. We consider $\mathbb{k}[\mathbb{N} A]$ as a subring of the Laurent polynomial ring $\mathbb{k}\left[y_{1}^{ \pm 1}, \ldots, y_{d}^{ \pm 1}\right]$. Then the ring of differential operators $D_{A}$ on the toric variety $X$ can be represented as a subring of the linear partial differential operators on $\partial_{y_{1}}, \ldots, \partial_{y_{d}}$ with Laurent polynomial coefficients,

$$
D_{A}=\bigoplus_{u \in \mathbb{Z}^{d}} y^{u}\left\{f(\theta) \in \mathbb{k}\left[\theta_{1}, \ldots, \theta_{d}\right] \mid f \text { vanishes on } \mathbb{N} A \backslash(-u+\mathbb{N} A)\right\}
$$

where $\theta_{1}, \ldots, \theta_{d}$ are the commuting operators $y_{1} \partial_{y_{1}}, \ldots, y_{d} \partial_{y_{d}}$.

3. The linear map $F: \mathbb{R}^{d} \rightarrow \mathbb{R}^{\mathscr{F}}$ induces a ring homomorphism

$$
\mathbb{k}[\mathbb{N} A] \rightarrow \mathbb{k}\left[\mathbb{N}^{\mathscr{F}}\right] \quad \text { via } \quad \sum_{j=1}^{k} \lambda_{j} y^{\beta_{j}} \mapsto \sum_{j=1}^{k} \lambda_{j} x^{F\left(\beta_{j}\right)},
$$

where $\beta_{1}, \ldots, \beta_{j} \in \mathbb{N} A$ and $\left(x_{\sigma}\right)_{\sigma \in \mathscr{F}}$ denote algebraically independent variables, so that $\mathbb{k}\left[\mathbb{N}^{\mathscr{F}}\right]=\mathbb{k}\left[x_{\sigma} \mid \sigma \in \mathscr{F}\right]$. For an ideal $I$ in $\mathbb{k}[\mathbb{N} A] \subset \mathbb{k}\left[y_{1}^{ \pm 1}, \ldots, y_{d}^{ \pm 1}\right]$, we abuse notation to denote

$$
F(I)=\left\{\sum \lambda_{j} x^{F\left(\beta_{j}\right)} \in \mathbb{k}\left[\mathbb{N}^{\mathscr{F}}\right] \mid \sum_{j} \lambda_{j} y^{\beta_{j}} \in I\right\} .
$$

Note that $F(I)$ is an ideal in the semigroup ring $\mathbb{k}[F(\mathbb{N} A)]$. The ideal in $\mathbb{k}[\mathbb{N} \mathscr{F}]$ generated by $F(I)$ is denoted by $J=\langle F(I)\rangle$.

The first goal of this paper is to extend the notion of $b$-functions to the toric setting. We use the same definition as in [BMS06a] except that the Weyl algebra $D$ is replaced by the ring of differential operators $D_{A}$ on $X$. Analyzing the combinatorics of the toric algebras, we obtain the following result.

Theorem (Theorem 3.4). The b-function $b_{I}(s)$ of an ideal I on the toric variety $X$ coincides with the b-function $b_{J}(s)$ of the ideal $J=\langle F(I)\rangle$ in the polynomial ring $\mathbb{k}\left[\mathbb{N}^{\mathscr{F}}\right]$. 
In particular, avoiding the study of the $V$-filtration in the toric case, we can still conclude that the toric $b$-function $b_{I}(s)$ is independent of the choices of generators of $I$.

To generalize Theorem 1.1 to the toric setting, we further restrict to the case where $I$ is a monomial ideal (i.e. an ideal defining a torus-invariant subvariety of $X$ ). In this case, the multiplier ideals of the pair $(X, I)$ were described in [Bli04], generalizing the work in [How01] on the multiplier ideals of monomial ideals in polynomial algebras. Using these formulas, we obtain a new expression of the multiplier ideals of monomial ideals on toric varieties using the map $F$ (Proposition 4.3),

$$
\mathcal{J}(X, \alpha I)=\left\langle y^{v} \in \mathbb{k}[\mathbb{N} A] \mid F(v)+e \in \operatorname{int}\left(\alpha P_{J}\right)\right\rangle,
$$

where $e \in \mathbb{N}^{\mathscr{F}}$ is such that $e_{\sigma}=1$ for all $\sigma \in \mathscr{F}$ and $\operatorname{int}\left(P_{J}\right)$ is the relative interior of the Newton polyhedron $P_{J}$ of the monomial ideal $J$ in $\mathbb{k}\left[\mathbb{N}^{\mathscr{F}}\right]$. In particular, we obtain the identity,

$$
\mathcal{J}(X, \alpha I)=\mathbb{k}[\mathbb{N} A] \cap \mathcal{J}\left(\mathbb{k}^{\mathscr{F}}, \alpha J\right)
$$

which relates the jumping coefficients of the pair $(X, I)$ to those of $\left(\mathbb{k}^{\mathscr{F}}, J\right)$. Since the jumping coefficients of $\left(\mathbb{K}^{\mathscr{F}}, J\right)$ are related to the roots of $b_{J}(-s)$ by Theorem [1.1, our arguments give the following theorem.

Theorem (Theorem 4.4). Let I be a monomial ideal on an affine normal toric variety $X$ over an algebraically closed field $\mathbb{k}$ of characteristic 0 . Then the log-canonical threshold of the pair $(X, I)$ coincides with the smallest root $\alpha_{I}$ of the b-function $b_{I}(-s)$, and any jumping coefficients of $(X, I)$ in $\left[\alpha_{I}, \alpha_{I}+1\right)$ are roots of $b_{I}(-s)$.

\section{Bernstein-Sato Polynomials of Budur, Mustaţă, and Saito}

We recall relevant background on Bernstein-Sato polynomials for ideals from the work of Budur, Mustaţă and Saito [BMS06a]. Here we concentrate on the case of subvarieties of affine spaces, but the definition works for subvarieties of a smooth variety.

Let $I$ be an ideal in $\mathbb{k}\left[x_{1}, \ldots, x_{n}\right]$, generated by $f_{1}, \ldots, f_{r}$. Denote by $D$ the Weyl algebra on $x_{1}, \ldots, x_{n}$. If $s_{1}, \ldots, s_{r}$ are indeterminates, consider

$$
\mathbb{k}\left[x_{1}, \ldots, x_{n}\right]\left[\prod_{i=1}^{r} f_{i}^{-1}, s_{1}, \ldots, s_{r}\right] \prod_{i=1}^{r} f_{i}^{s_{i}} .
$$

This is a $D\left[s_{i j}\right]$-module, where $s_{i j}=s_{i} t_{i}^{-1} t_{j}$, and the action of the operator $t_{i}$ is given by $t_{i}\left(s_{j}\right)=$ $s_{j}+\delta_{i j}$ (the Kronecker delta).

Definition 2.1. The Bernstein-Sato polynomial or b-function associated to $f=\left(f_{1}, \ldots, f_{r}\right)$ is defined to be the monic polynomial of the lowest degree in $s=\sum_{i=1}^{r} s_{i}$ satisfying a relation of the form

$$
b_{f}(s) \prod_{i=1}^{r} f_{i}^{s_{i}}=\sum_{k=1}^{r} P_{k} t_{k} \prod_{i=1}^{r} f_{i}^{s_{i}}
$$

where $P_{1}, \ldots, P_{r} \in D\left[s_{i j} \mid i, j \in\{1, \ldots, r\}\right]$.

In [BMS06a, Section 2], Budur, Mustaţă and Saito show that:

1. $b_{f}(s)$ is a nonzero polynomial,

2. $b_{f}(s)$ is independent of the generating set of $I$. 
2.1. An alternative way to define the Bernstein-Sato polynomial. In [BMS06a, Section 2.10] Budur, Mustaţă and Saito give the following way to compute the $b$-function.

For $c=\left(c_{1}, \ldots, c_{r}\right) \in \mathbb{Z}^{r}$, define $\operatorname{nsupp}(c)=\left\{i \mid c_{i}<0\right\}$. The Bernstein-Sato polynomial $b_{f}(s)$ is the monic polynomial of the smallest degree such that $b_{f}(s) \prod_{i=1}^{r} f_{i}^{s_{i}}$ belongs to the $D\left[s_{1}, \ldots, s_{r}\right]$-submodule generated by

$$
\prod_{i \in \operatorname{nsupp}(c)}\left(\begin{array}{c}
s_{i} \\
-c_{i}
\end{array}\right) \cdot \prod_{i=1}^{r} f_{i}^{s_{i}+c_{i}}
$$

where $c=\left(c_{1}, \ldots, c_{r}\right)$ runs over the elements of $\mathbb{Z}^{r}$ such that $\sum_{i=1}^{r} c_{i}=1$. Here $s=\sum_{i=1}^{r} s_{i}$ and $\left(\begin{array}{c}s_{i} \\ m\end{array}\right)=s_{i}\left(s_{i}-1\right) \cdots\left(s_{i}-m+1\right) / m$ !.

The advantage of this approach is that one now works in the ring $D\left[s_{1}, \ldots, s_{r}\right]$, and need no longer consider the operators $s_{i j}$ or $t_{k}$.

2.2. A monomial-ideal-specific way to define the Bernstein-Sato polynomial. The definition given in the previous subsection can be further specialized to the case of monomial ideals.

Let $\mathfrak{a}$ be a monomial ideal in $\mathbb{k}\left[x_{1}, \ldots, x_{n}\right]$ whose minimal monomial generators are $x^{\alpha_{1}}, \ldots, x^{\alpha_{r}}$. Set $\ell_{\alpha}\left(s_{1}, \ldots, s_{r}\right)=s_{1} \alpha_{1}+\cdots+s_{r} \alpha_{r}$, and $\ell_{i}(s)$ to be the $i$ th coordinate of this vector. For $v \in \mathbb{Z}^{r}$, let $\operatorname{psupp}(v)=\left\{i \mid v_{i}>0\right\}$.

For $c=\left(c_{1}, \ldots, c_{r}\right) \in \mathbb{Z}^{r}$ such that $\sum_{i=1}^{r} c_{i}=1$, set

$$
g_{c}\left(s_{1}, \ldots, s_{r}\right)=\prod_{j \in \operatorname{nsupp}(c)}\left(\begin{array}{c}
s_{j} \\
-c_{j}
\end{array}\right) \cdot \prod_{i \in \operatorname{psupp}\left(\ell_{\alpha}(c)\right)}\left(\begin{array}{c}
\ell_{i}\left(s_{1}, \ldots, s_{r}\right)+\ell_{i}(c) \\
\ell_{i}(c)
\end{array}\right),
$$

and define $I_{\mathfrak{a}}$ to be the ideal of $\mathbb{Q}\left[s_{1}, \ldots, s_{r}\right]$ generated by the polynomials $g_{c}$.

Then [BMS06a, Proposition 4.2] asserts that $b_{f}(s)$ is the monic polynomial of smallest degree such that $b_{f}\left(\sum_{i=1}^{r} s_{i}\right)$ belongs to the ideal $I_{\mathfrak{a}}$.

\section{Bernstein-Sato Polynomials on Toric VARieties}

The goal of this section is to extend Definition 2.1 to the toric setting. We first recall some background on the ring of differential operators of a toric variety.

3.1. Rings of differential operators on toric varieties. For a commutative algebra $R$ over a commutative ring $k$, Grothendieck's ring of $k$-linear differential operators $D_{k}(R)$ of $R$ [Gro67] is the $R$-subalgebra of $\operatorname{Hom}_{k}(R, R)$ defined by

$$
D_{k}(R)=\bigcup_{i \in \mathbb{N} \cup\{0\}} D_{i}
$$

where $D_{0}=R$ and $D_{i}=\left\{f \in \operatorname{Hom}_{k}(R, R) \mid f r-r f \in D_{i-1}\right.$ for all $\left.r \in R\right\}$ for $i \geq 1$. When $R$ is the polynomial ring of $n$ variables over an algebraically closed field $k$ of characteristic 0 , the ring $D_{k}(R)$ coincides with the $n$th Weyl algebra. Moreover, it is well known that if $R$ is regular over $k$, then $D_{k}(R)$ is the $R$-algebra generated by the $k$-derivations of $R$. The most influential applications of $D$-module theory, and in particular, the $b$-functions of [BMS06a], are built in this setting. 
When $R$ is not regular, the ring $D_{k}(R)$ is not well-behaved [BGG72]. Nonetheless, when $R=$ $\mathbb{k}[\mathbb{N} A]$ is a toric algebra over an algebraically closed field $\mathbb{k}$ of characteristic 0 , there exists an explicit combinatorial description of $D_{\mathbb{k}}(\mathbb{k}[\mathbb{N} A])$. This expression of $D_{\mathbb{k}}(\mathbb{k}[\mathbb{N} A])$ was obtained independently in [Mus87] and [Jon94] when $\mathbb{N} A$ is normal, and was further extended to the not necessarily normal case in [ST01]. We denote the ring of differential operators of the toric algebra $\mathbb{k}[\mathbb{N} A]$ by

$$
D_{A}=D_{\mathbb{k}}(\mathbb{k}[\mathbb{N} A]) .
$$

One way to understand $D_{A}$ is to identify $\mathbb{k}[\mathbb{N} A]$ as a subring of the Laurent polynomial ring $\mathbb{k}\left[y_{1}^{ \pm 1}, \ldots, y_{d}^{ \pm 1}\right]$ and study the $\mathbb{k}$-linear differential operators of $\mathbb{k}\left[y_{1}^{ \pm 1}, \ldots, y_{d}^{ \pm 1}\right]$. It is known that if $S$ is a multiplicatively closed subset of a $k$-algebra $R$, then $D_{k}\left(S^{-1} R\right) \cong S^{-1} R \otimes_{R} D_{k}(R)$. It follows that

$$
D_{\mathbb{k}}\left(\mathbb{k}\left[y_{1}^{ \pm 1}, \ldots, y_{d}^{ \pm 1}\right]\right)=\mathbb{k}\left[y_{1}^{ \pm 1}, \ldots, y_{d}^{ \pm 1}\right]\left\langle\partial_{y_{1}}, \ldots, \partial_{y_{d}}\right\rangle,
$$

is a localization of the $n$th Weyl algebra. Moreover, one can realize

$$
D_{A}=\left\{\delta \in D_{\mathbb{k}}\left(\mathbb{k}\left[y_{1}^{ \pm 1}, \ldots, y_{d}^{ \pm 1}\right]\right) \mid \delta(\mathbb{k}[\mathbb{N} A]) \subseteq \mathbb{k}[\mathbb{N} A]\right\}
$$

as a subring of $D_{\mathbb{k}}\left(\mathbb{k}\left[y_{1}^{ \pm 1}, \ldots, y_{d}^{ \pm 1}\right]\right)$. In particular, we have the $\mathbb{Z}^{d}$-graded expression

$$
D_{A}=\bigoplus_{u \in \mathbb{Z}^{d}} y^{u}\left\{f(\theta) \in \mathbb{k}\left[\theta_{1}, \ldots, \theta_{d}\right] \mid f \text { vanishes on } \mathbb{N} A \backslash(-u+\mathbb{N} A)\right\}
$$

where $\theta_{1}, \ldots, \theta_{d}$ are the commuting operators $y_{i} \partial_{y_{i}}$, for $i=1, \ldots, d$. Since we are only concerned with the case when $\mathbb{N} A$ is normal, for a given $u \in \mathbb{Z}^{d}$, the ideal

$$
\left\{f(\theta) \in \mathbb{k}\left[\theta_{1}, \ldots, \theta_{d}\right] \mid f \text { vanishes on } \mathbb{N} A \backslash(-u+\mathbb{N} A)\right\}
$$

is the principal ideal in $\mathbb{C}\left[\theta_{1}, \ldots, \theta_{d}\right]$ generated by

$$
\prod_{F_{\sigma}(u)>0} \prod_{j=0}^{F_{\sigma}(u)-1}\left(F_{\sigma}\left(\theta_{1}, \ldots, \theta_{d}\right)-j\right),
$$

where the product runs through all facets $\sigma$ (with $F_{\sigma}(u)>0$ ) of the cone $C$ associated to $\mathbb{N} A$, and $F_{\sigma}$ is the primitive integral support function of $\sigma$ as defined in Notation 1.2]1.

We are now ready to define the $b$-function for an ideal $I$ in $\mathbb{k}[\mathbb{N} A]$. To make the exposition more transparent, we first treat the case when $I$ is an monomial ideal.

3.2. Monomial ideals in semigroup rings. Let $\mathfrak{a}$ be a monomial ideal in $\mathbb{k}[\mathbb{N} A]$ whose minimal monomial generators are Laurent monomials $y^{\beta_{1}}, \ldots, y^{\beta_{r}}$. For $f=\left(y^{\beta_{1}}, \ldots, y^{\beta_{r}}\right)$, we define the Bernstein-Sato polynomial $b_{f}(s)$ exactly as in Definition 2.1, except that the Weyl algebra $D$ is replaced by the ring of differential operators $D_{A}$ on $\mathbb{k}[\mathbb{N} A]$.

We note that the reduction in Subsection 2.1 goes unchanged when we switch to the setting of semigroup rings, since that reduction is concerned only with the operators $s_{i j}$ and $t_{k}$, and does not depend at all on the ambient differential operators.

Our task becomes to translate the situation from Subsection 2.2 to the new setting with monomial ideals in semigroup rings, rather than monomial ideals in polynomial rings.

Let $c \in \mathbb{Z}^{r}$ whose coordinates sum to 1 , and for $\beta=\left(\beta_{1}, \ldots, \beta_{r}\right) \in\left(\mathbb{Z}^{d}\right)^{r}$, set $\ell_{\beta}\left(s_{1}, \ldots, s_{r}\right)=$ $s_{1} \beta_{1}+\cdots+s_{r} \beta_{r}$. 
In order to find the Bernstein-Sato polynomial, we need to apply an operator in $D_{A}\left[s_{1}, \ldots, s_{r}\right]$ to $\prod_{i \in \operatorname{nsupp}(c)}\left(\begin{array}{c}s_{1} \\ -c_{i}\end{array}\right) \cdot \prod_{i=1}^{r}\left(y^{\beta_{i}}\right)^{s_{i}+c_{i}}$ in such a way that the outcome is a multiple of $y^{\sum_{i=1}^{r} s_{i} \beta_{i}}=$ $y^{\ell_{\beta}\left(s_{1}, \ldots, s_{r}\right)}$.

If we apply the operator from (3.2) with $u=\ell_{\beta}(c)$ to $\prod_{i \in \operatorname{nsupp}(c)}\left(\begin{array}{c}s_{1} \\ -c_{i}\end{array}\right) \cdot \prod_{i=1}^{r}\left(y^{\beta_{i}}\right)^{s_{i}+c_{i}}$ we obtain

$$
\prod_{i \in \operatorname{nsupp}(c)}\left(\begin{array}{c}
s_{1} \\
-c_{i}
\end{array}\right) \prod_{F_{\sigma}(\ell(c))>0} \prod_{j=0}^{F_{\sigma}(\ell(c))-1}\left(F_{\sigma}\left(\ell_{\beta}\left(s_{1}, \ldots, s_{r}\right)+\ell_{\beta}(c)\right)-j\right) \cdot y^{\ell\left(s_{1}, \ldots, s_{r}\right)} .
$$

Proposition 3.1. We use the notation introduced above. The Bernstein-Sato polynomial $b_{f}(s)$ for $f=\left(y^{\beta_{1}}, \ldots, y^{\beta_{r}}\right)$ considered as a monomial ideal in $\mathbb{k}[\mathbb{N} A]$ is the monic polynomial of smallest degree such that $b_{f}\left(s_{1}+\cdots+s_{r}\right)$ belongs to the ideal generated by

$$
\prod_{i \in \operatorname{nsupp}(c)}\left(\begin{array}{c}
s_{1} \\
-c_{i}
\end{array}\right) \prod_{F_{\sigma}\left(\ell_{\beta}(c)\right)>0}\left(\begin{array}{c}
F_{\sigma}\left(\ell_{\beta}\left(s_{1}, \ldots, s_{r}\right)+\ell_{\beta}(c)\right) \\
F_{\sigma}\left(\ell_{\beta}(c)\right)
\end{array}\right)
$$

where $c \in \mathbb{Z}^{r}$ has coordinate sum 1 .

Proof. It remains to be shown that, for any other operator (in $D_{A}\left[s_{1}, \ldots, s_{r}\right]$ ) which applied to $\prod_{i \in \operatorname{nsupp}(c)}\left(\begin{array}{c}s_{1} \\ -c_{i}\end{array}\right) \cdot \prod_{i=1}^{r}\left(y^{\beta_{i}}\right)^{s_{i}+c_{i}}$ yields a multiple of $y^{\ell\left(s_{1}, \ldots, s_{r}\right)}$, this multiple belongs to the ideal generated by (3.3).

This follows from the description of $D_{A}$ given by (3.1) and (3.2).

Recall that $\mathscr{F}$ is the collection of facets of the cone $C$ of $\mathbb{N} A$, and that the map $F: \mathbb{R}^{d} \rightarrow \mathbb{R}^{\mathscr{F}}$ defined by $F(p)=\left(F_{\sigma}(p)\right)_{\sigma \in \mathscr{F}}$ from Notation 1.211 induces an inclusion $\mathbb{k}[\mathbb{N} A] \rightarrow \mathbb{k}\left[\mathbb{N}^{\mathscr{F}}\right]$.

Proposition 3.2. Denote by $F(f)$ the sequence of monomials $\left(x^{F\left(\beta_{1}\right)}, \ldots, x^{F\left(\beta_{r}\right)}\right)$ in $\mathbb{k}\left[\mathbb{N}^{\mathscr{F}}\right]$. The Bernstein-Sato polynomial $b_{F(f)}(s)$ of $F(f)$ in the polynomial ring $\mathbb{k}\left[\mathbb{N}^{\mathscr{F}}\right]$ coincides with the Bernstein-Sato polynomial $b_{f}(s)$ of $f=\left(x^{\beta_{1}}, \ldots, x^{\beta_{r}}\right)$ in $\mathbb{k}[\mathbb{N} A]$. In particular, the latter polynomial $b_{f}(s)$ is nonzero, and its roots can be computed using the combinatorial description [BMS06b, Theorem 1.1] applied to $\left\langle x^{F\left(\beta_{1}\right)}, \ldots, x^{F\left(\beta_{r}\right)}\right\rangle \subseteq \mathbb{k}\left[\mathbb{N}^{\mathscr{F}}\right]$.

Proof. First we observe that the minimal monomial generators of $\left\langle x^{F\left(\beta_{1}\right)}, \ldots, x^{F\left(\beta_{r}\right)}\right\rangle \subseteq \mathbb{k}^{\left[\mathbb{N}^{\mathscr{F}}\right]}$ are the monomials $x^{F\left(\beta_{1}\right)}, \ldots, x^{F\left(\beta_{r}\right)}$. To see this, note that $x^{F\left(\beta_{i}\right)}$ divides $x^{F\left(\beta_{j}\right)}$ is equivalent to $F_{\sigma}\left(\beta_{i}\right) \leq F_{\sigma}\left(\beta_{j}\right)$ for all facets $\sigma$ of the cone $C$. This implies that $\beta_{j}-\beta_{i}$ lies in the cone $C$. Since $\beta_{j}-\beta_{i} \in \mathbb{Z}^{r}$ and $\mathbb{N} A$ is normal, we see that $\beta_{j}-\beta_{i} \in \mathbb{N} A$, and therefore $y^{\beta_{i}}$ divides $y^{\beta_{j}}$ in $\mathbb{k}[\mathbb{N} A]$. Let $\alpha_{i}=F\left(\beta_{i}\right)$ for $i=1, \ldots, r$, and $\ell_{\alpha}\left(s_{1}, \ldots, s_{r}\right)=s_{1} \alpha_{1}+\cdots+s_{r} \alpha_{r}$, and denote by $\ell_{i}\left(s_{1}, \ldots, s_{r}\right)$ the $i$ th coordinate of the vector $\ell_{\alpha}\left(s_{1}, \ldots, s_{r}\right)$. By [BMS06a, Proposition 4.2] the Bernstein-Sato polynomial of $\left\langle x^{F\left(\beta_{1}\right)}, \ldots, x^{F\left(\beta_{r}\right)}\right\rangle$ is the monic polynomial $p$ of smallest degree such that $p\left(s_{1}+\cdots+s_{r}\right)$ lies in the ideal generated by

$$
\prod_{j \in \operatorname{nsupp}(c)}\left(\begin{array}{c}
s_{j} \\
-c_{j}
\end{array}\right) \prod_{i \in \operatorname{psupp}\left(\ell_{\alpha}(c)\right)}\left(\begin{array}{c}
\ell_{i}\left(s_{1}, \ldots, s_{r}\right)+\ell_{i}(c) \\
\ell_{i}(c)
\end{array}\right)
$$

for $c \in \mathbb{Z}^{r}$ with coordinate sum 1 . But note that by construction $\ell_{\alpha}=F \circ \ell_{\beta}$, so that the generators (3.4) coincide exactly with the generators (3.3). As a reality check, note that both sets of generators belong to the ring $\mathbb{k}\left[s_{1}, \ldots, s_{r}\right]$, which does not depend on the ambient rings of the monomial ideals involved. 
3.3. The general case. Again, recall that we have a linear map $F: \mathbb{R}^{d} \rightarrow \mathbb{R}^{\mathscr{F}}$ with $F(\mathbb{N} A) \subseteq \mathbb{N}^{\mathscr{F}}$. We construct a ring homomorphism

$$
\mathbb{k}[\mathbb{N} A] \rightarrow \mathbb{k}\left[\mathbb{N}^{\mathscr{F}}\right] \quad \text { via } \quad \sum_{j=1}^{k} \lambda_{j} y^{\beta_{j}} \mapsto \sum_{j=1}^{k} \lambda_{j} x^{F\left(\beta_{j}\right)},
$$

where $\beta_{1}, \ldots, \beta_{j} \in \mathbb{N} A$. That this is a homomorphism follows from linearity of $F$, as $x^{F\left(\beta+\beta^{\prime}\right)}=$ $x^{F(\beta)+F\left(\beta^{\prime}\right)}=x^{F(\beta)} x^{F\left(\beta^{\prime}\right)}$. We abuse notation and denote the homomorphism (3.5) by $F$.

If $I \subset \mathbb{k}[\mathbb{N} A]$ is an ideal, then its image $F(I)$ is not an ideal in $\mathbb{k}\left[\mathbb{N}^{\mathscr{F}}\right]$; we denote by $\langle F(I)\rangle$ the ideal in $\mathbb{k}\left[\mathbb{N}^{\mathscr{F}}\right]$ generated by $F(I)$.

Lemma 3.3. Let $I \subset \mathbb{k}[\mathbb{N} A]$ be an ideal generated by $g_{1}, \ldots, g_{r} \in \mathbb{k}[\mathbb{N} A]$. Then $F\left(g_{1}\right), \ldots, F\left(g_{r}\right)$ generate $\langle F(I)\rangle$.

Proof. Since $g_{1}, \ldots, g_{r}$ generate $I$, and $F$ is a ring homomorphism, any element of $F(I)$ is obtained as a combination of $F\left(g_{1}\right), \ldots, F\left(g_{r}\right)$ with coefficients in $\mathbb{k}\left[\mathbb{N}^{\mathscr{F}}\right]$. This implies that the polynomials $F\left(g_{1}\right), \ldots, F\left(g_{r}\right)$ generate $\langle F(I)\rangle$.

We apply Definition 2.1 to a sequence of polynomials in $\mathbb{k}[\mathbb{N} A]$, using the ring of differential operators $D_{A}$ instead of $D$. The following is the main result of this section.

Theorem 3.4. Let $I \subset \mathbb{k}[\mathbb{N} A]$ be an ideal, and let $g_{1}, \ldots, g_{r}$ be generators for $I$. The BernsteinSato polynomial of $g=\left(g_{1}, \ldots, g_{r}\right)$ in $\mathbb{k}[\mathbb{N} A]$ equals the Bernstein-Sato polynomial of $f=$ $\left(f_{1}, \ldots, f_{r}\right)=F(g)=\left(F\left(g_{1}\right), \ldots, F\left(g_{r}\right)\right)$ in $\mathbb{k}\left[\mathbb{N}^{\mathscr{F}}\right]$. Consequently, this Bernstein-Sato polynomial is nonzero, and depends only on $I$, not on the particular set of generators chosen.

Proof. We know that the Bernstein-Sato polynomial $b_{g}(s)$ is the monic polynomial of smallest degree such that, for $s=s_{1}+\cdots+s_{r}, b_{g}(s) \prod_{i=1}^{r} g_{i}^{s_{i}}$ belongs to the $D_{A}\left[s_{1}, \ldots, s_{r}\right]$-submodule generated by

$$
\prod_{i \in \operatorname{nsupp}(c)}\left(\begin{array}{c}
s_{i} \\
-c_{i}
\end{array}\right) \cdot \prod_{i=1}^{r} g_{i}^{s_{i}+c_{i}}, \quad \text { for }\left(c_{1}, \ldots, c_{r}\right) \in \mathbb{Z}^{r} \text { with } \sum_{i=1}^{r} c_{i}=1,
$$

while $b_{f}(s)$ is is the monic polynomial of smallest degree such that, $b_{f}(s) \prod_{i=1}^{r} f_{i}^{s_{i}}$ belongs to the $D\left[s_{1}, \ldots, s_{r}\right]$-submodule generated by

$$
\prod_{i \in \operatorname{nsupp}(c)}\left(\begin{array}{c}
s_{i} \\
-c_{i}
\end{array}\right) \cdot \prod_{i=1}^{r} f_{i}^{s_{i}+c_{i}}, \quad \text { for }\left(c_{1}, \ldots, c_{r}\right) \in \mathbb{Z}^{r} \text { with } \sum_{i=1}^{r} c_{i}=1 .
$$

Here $D$ is the ring of differential operators on $\mathbb{k}\left[\mathbb{N}^{\mathscr{F}}\right]$, and $D_{A}$ is the ring of differential operators on $\mathbb{k}[\mathbb{N} A]$.

Fix $c \in \mathbb{Z}^{r}$ such that $\sum c_{i}=1$, and let $P \in D\left[s_{1}, \ldots, s_{r}\right]$ such that $P\left[\prod_{i \in \operatorname{nsupp}(c)}\left(\begin{array}{c}s_{i} \\ -c_{i}\end{array}\right)\right.$. $\left.\prod_{i=1}^{r} f_{i}^{s_{i}+c_{i}}\right]$ is a polynomial in $s_{1}, \ldots, s_{r}$ times $\prod_{i=1}^{r} f_{i}^{s_{i}}$. Applying the description given by (3.1) and (3.2) to $D$ (instead of $D_{A}$ ), we see that we can write $P$ as a finite sum

$$
P=\sum_{u \in \mathbb{Z}^{\mathscr{F}}} q_{u}\left(s_{1}, \ldots, s_{r}\right) x^{u}\left[\prod_{u_{\sigma}<0} \prod_{j=0}^{-u_{\sigma}-1}\left(\left(\theta_{x}\right)_{\sigma}-j\right)\right] p_{u}\left(\theta_{x}\right),
$$

where the $p_{u}$ are polynomials in $|\mathscr{F}|$ indeterminates with coefficients in $\mathbb{k}$. 
Note that, by construction, the element $\prod_{i \in \operatorname{nsupp}(c)}\left(\begin{array}{c}s_{i} \\ -c_{i}\end{array}\right) \cdot \prod_{i=1}^{r} f_{i}^{s_{i}+c_{i}}$ is $F(\mathbb{N} A)$-graded. Since $P\left[\prod_{i \in \operatorname{nsupp}(c)}\left(\begin{array}{c}s_{i} \\ -c_{i}\end{array}\right) \cdot \prod_{i=1}^{r} f_{i}^{s_{i}+c_{i}}\right]$ is a multiple of $\prod_{i=1}^{r} f_{i}^{s_{i}}$, we may assume that the operator $P$ is $F(\mathbb{N} A)$-graded as well. In other words, we may assume that $u \in F\left(\mathbb{Z}^{d}\right)$ if $q_{u} p_{u} \neq 0$.

Thus we rewrite

$$
P=\sum_{u=F(v) \in F\left(\mathbb{Z}^{d}\right)} q_{u}\left(s_{1}, \ldots, s_{r}\right) x^{u}\left[\prod_{u_{\sigma}<0} \prod_{j=0}^{-u_{\sigma}-1}\left(\left(\theta_{x}\right)_{\sigma}-j\right)\right] p_{u}\left(\theta_{x}\right),
$$

and if we denote

$$
\hat{P}=\sum_{v \in \mathbb{Z}^{d}} q_{F(v)}\left(s_{1}, \ldots, s_{r}\right) y^{v}\left[\prod_{F_{\sigma}(v)<0} \prod_{j=0}^{-F_{\sigma}(v)-1}\left(F_{\sigma}\left(\theta_{y}\right)-j\right)\right] p_{F(v)}\left(\left(F_{\sigma}\left(\theta_{y}\right)\right)_{\sigma \in \mathscr{F}}\right),
$$

then $\hat{P}$ is an element of $D_{A}\left[s_{1}, \ldots, s_{r}\right]$, and $\hat{P}$ applied to $\prod_{i \in \operatorname{nsupp}(c)}\left(\begin{array}{c}s_{i} \\ -c_{i}\end{array}\right) \cdot \prod_{i=1}^{r} g_{i}^{s_{i}+c_{i}}$ is a polynomial of $s_{1}, \ldots, s_{r}$ times $\prod_{i=1}^{r} g_{i}^{s_{i}}$, and this is the same polynomial that we obtain when we apply $P$ to $\prod_{i \in \operatorname{nsupp}(c)}\left(\begin{array}{c}s_{i} \\ -c_{i}\end{array}\right) \cdot \prod_{i=1}^{r} f_{i}^{s_{i}+c_{i}}$ (and divide by $\prod f_{i}^{s_{i}}$ ).

Conversely, if we apply an element of $D_{A}\left[s_{1}, \ldots, s_{r}\right]$ to $\prod_{i \in \operatorname{nsupp}(c)}\left(\begin{array}{c}s_{i} \\ -c_{i}\end{array}\right) \cdot \prod_{i=1}^{r} g_{i}^{s_{i}+c_{i}}$ and obtain a polynomial in $s_{1}, \ldots, s_{r}$ times $\prod_{i=1}^{r} g_{i}^{s_{i}}$, then we can obtain an element of $D\left[s_{1}, \ldots, s_{r}\right]$ which applied to $\prod_{i \in \operatorname{nsupp}(c)}\left(\begin{array}{c}s_{i} \\ -c_{i}\end{array}\right) \cdot \prod_{i=1}^{r} f_{i}^{s_{i}+c_{i}}$ gives exactly the same polynomial in $s_{1}, \ldots, s_{r}$ times $\prod_{i=1}^{r} f_{i}^{s_{i}}$. To do this, we write the element of $D_{A}\left[s_{1}, \ldots, s_{r}\right]$ as a sum of terms of the form

$$
q_{v}\left(s_{1}, \ldots, s_{r}\right) y^{v}\left[\prod_{F_{\sigma}(v)<0} \prod_{j=0}^{-F_{\sigma}(v)-1}\left(F_{\sigma}\left(\theta_{y}\right)-j\right)\right] p_{v}\left(\theta_{y}\right),
$$

then express (non uniquely) the polynomial $p_{v}$ as sums of powers of the linear forms $F_{\sigma}\left(\theta_{y}\right)$, and then apply $F$ in the obvious way. Nonuniqueness comes because we need to choose $d$ linearly independent forms $F_{\sigma}$ in order to get $\mathbb{k}$-algebra generators of $\mathbb{k}\left[\theta_{y}\right]$; however, since the image of $F$ as a linear map is $d$-dimensional, the choice does not affect the image.

\section{Multiplier Ideals on Normal TORIC VARIETIES}

We recall basic definitions of multiplier ideals that can be found in Lazarsfeld's text, Positivity in Algebraic Geometry II [Laz04].

Let $X$ be a smooth variety over an algebraically closed field $\mathbb{k}$ of characteristic 0 . Let $\mathcal{I} \subseteq \mathcal{O}_{X}$ be an ideal sheaf, and $\alpha>0$ a rational number. Fix a $\log$ resolution $\mu: X^{\prime} \rightarrow X$ of $\mathcal{I}$ with $\mathcal{I} \cdot \mathcal{O}_{X^{\prime}}=\mathcal{O}_{X^{\prime}}(-E)$. The multiplier ideal of the pair $(X, \alpha \mathcal{I})$ is defined as

$$
\mathcal{J}(X, \alpha \mathcal{I})=\mu_{*} \mathcal{O}_{X^{\prime}}\left(K_{X^{\prime} / X}-\lfloor\alpha \cdot E\rfloor\right),
$$

where $K_{X^{\prime} / X}=K_{X^{\prime}}-\mu^{*} K_{X}$ is the relative canonical divisor of $\mu$, which is an effective divisor supported on the exceptional locus of $\mu$ whose local equation is given by the determinant of the derivative $d \mu$. The definition does not depend on the choice of log resolution. One of the important features of multiplier ideals is that $\mathcal{J}(X, \alpha \mathcal{I})$ measures the singularity of the pair $(X, \alpha \mathcal{I})$ : a smaller multiplier ideal corresponds to a worse singularity.

Notice that $\mathcal{J}(X, \alpha \mathcal{I})$ becomes smaller as $\alpha$ increases. The jumping coefficients of $(X, \alpha \mathcal{I})$ are the positive real numbers $0<\alpha_{1}<\alpha_{2}<\ldots$ such that $\mathcal{J}\left(X, \alpha_{j} \mathcal{I}\right)=\mathcal{J}(X, \alpha \mathcal{I}) \neq \mathcal{J}\left(X, \alpha_{j+1} \mathcal{I}\right)$ for $\alpha_{j} \leq \alpha<\alpha_{j+1}(j \geq 0)$ where $\alpha_{0}=0$. When $X$ is affine and $I$ is the ideal in the coordinate ring 
$\mathbb{k}[X]$ corresponding the the sheaf $\mathcal{I}$, Budur, Mustaţă and Saito proved that jumping coefficients of $(X, \alpha I)$ in $\left[\alpha_{f}, \alpha_{f}+1\right)$ are roots of $b_{f}(-s)$, where $f=\left(f_{1}, \ldots, f_{r}\right)$ is a set of generators for $I$, $b_{f}(s)$ is the Bernstein-Sato polynomial of $I$, and $\alpha_{f}$ is the smallest root of $b_{f}(-s)$. One of our goals is to generalize this correspondence between $b$-function roots and jumping coefficients to monomial ideals on affine normal toric varieties.

In the special case that $I$ is a monomial ideal in the polynomial ring $\mathbb{k}\left[x_{1}, \ldots, x_{m}\right]$, Howald gave the following combinatorial formula for multiplier ideal of the pair $\left(\mathbb{A}^{m}, \alpha I\right)$,

$$
\mathcal{J}\left(\mathbb{A}^{m}, \alpha I\right)=\left\langle x^{v} \mid v+e \in \operatorname{int}\left(\alpha P_{I}\right)\right\rangle,
$$

where $P_{I}$ is the Newton polyhedron of $I$ and $e$ is the vector $(1,1, \ldots, 1)$ in $\mathbb{N}^{m}$. In particular, a rational number $\alpha>0$ is a jumping coefficient of $\left(\mathbb{A}^{m}, \alpha I\right)$ if and only if the boundary of $-e+\operatorname{int}\left(\alpha P_{I}\right)$ contains a lattice point in $\mathbb{N}^{m}$.

The notion of multiplier ideal can be generalized to the case that $\mathcal{I}$ is an ideal sheaf on a normal variety $X$ over $\mathbb{k}$ as follows. Let $\Delta$ be an effective $\mathbb{Q}$-divisor such that $K_{X}+\Delta$ is $\mathbb{Q}$-Cartier. Such $\Delta$ is called a boundary divisor. Let $\mu: X^{\prime} \rightarrow X$ be a log resolution of the triple $(X, \Delta, \mathcal{I})$ and $\alpha>0$ be a rational number. Suppose that $\mathcal{I} \cdot \mathcal{O}_{X^{\prime}}=\mathcal{O}_{X^{\prime}}(-E)$. Then one can define the multiplier ideal $\mathcal{J}(X, \Delta, \alpha \mathcal{I})$ associated to the triple $(X, \Delta, \alpha \mathcal{I})$ as

$$
\mathcal{J}(X, \Delta, \alpha \mathcal{I})=\mu_{*} \mathcal{O}_{X^{\prime}}\left(K_{X^{\prime}}-\left\lfloor\mu^{*}\left(K_{X}+\Delta\right)+\alpha E\right\rfloor\right) .
$$

Again, this definition is not dependent on the choice of $\mu$. De Fernex and Hacon [dFH09] have given a definition of $\mathcal{J}(X, \alpha Z)$ for non- $\mathbb{Q}$-Gorenstein $X$ without using the boundary divisor $\Delta$. They showed that there exists a boundary divisor $\Delta$ such that the multiplier ideal $\mathcal{J}(X, \Delta, \alpha Z)$ coincides with their multiplier ideal $\mathcal{J}(X, \alpha Z)$ and that $\mathcal{J}(X, \alpha Z)$ is the unique maximal element of the set

$$
\{\mathcal{J}(X, \Delta, \alpha Z) \mid \Delta \text { is a boundary divisor }\} .
$$

4.1. A New expression for multiplier ideals on toric varieties. Let us explain how one can use the map $F$ to understand the multiplier ideals of De Fernex and Hacon in the case of monomial ideals on normal toric varieties.

Let $\mathbb{k}[\mathbb{N} A] \subset \mathbb{k}\left[y_{1}^{ \pm}, \ldots, y_{d}^{ \pm}\right]$be a normal semigroup ring in our setting and let $X=\operatorname{Spec}(\mathbb{R}[\mathbb{N} A])$. Each facet $\sigma \in \mathscr{F}$ corresponds to a torus invariant prime Weil divisor $D_{\sigma}$ on $X$. The canonical class of $X$ is represented by the torus invariant canonical divisor $K_{X}=-\sum_{\sigma \in \mathscr{F}} D_{\sigma}$. A boundary divisor $\Delta$ is an effective $\mathbb{Q}$-divisor such that $K_{X}+\Delta$ is $\mathbb{Q}$-Cartier, which means there exist $l \in \mathbb{Z}$ and $u \in \mathbb{Z}^{d}$ such that $l\left(K_{X}+\Delta\right)=\operatorname{div}\left(y^{u}\right)$. Denote $w_{\Delta}=\frac{u}{l}$. Notice that

$$
\Delta=\sum_{\sigma \in \mathscr{F}}\left(1+F_{\sigma}\left(w_{\Delta}\right)\right) D_{\sigma}
$$

so the effectivity of $\Delta$ is equivalent to the condition that $F_{\sigma}\left(w_{\Delta}\right) \geq-1$ for all $\sigma \in \mathscr{F}$. Conversely, each $w \in \mathbb{Q}^{d}$ gives rise to a boundary divisor $\Delta_{w}$ on $X$ by the same formula

$$
\Delta_{w}=\sum_{\sigma \in \mathscr{F}}\left(1+F_{\sigma}(w)\right) D_{\sigma} .
$$

Let $I$ be a monomial ideal in $\mathbb{k}[\mathbb{N} A]$ and let $\alpha>0$ be a rational number. Blickle [Bli04] showed that the multiplier ideal $\mathcal{J}(X, \Delta, \alpha I)=\left\langle y^{v} \in \mathbb{k}[\mathbb{N} A] \mid v-w_{\Delta} \in \operatorname{int}\left(\alpha P_{I}\right)\right\rangle$, generalizing Howald's 
description of multiplier ideals [How01] in the case of monomial ideals in polynomial rings. Be cautioned about the mistake of the sign of $w_{\Delta}$ in Blickle's original description. For $w \in \mathbb{Q}^{d}$, denote

$$
\Omega_{w, \alpha I}=\left[w+\operatorname{int}\left(\alpha P_{I}\right)\right] \text { and } \Omega_{\alpha I}=\bigcup_{w \in \mathbb{Q}^{d}: F_{\sigma}(w) \geq-1 \forall \sigma \in \mathscr{F}} \Omega_{w, \alpha I} .
$$

Then the multiplier ideal of De Fernex and Hacon is

$$
\mathcal{J}(X, \alpha I)=\left\langle y^{v} \in \mathbb{k}[\mathbb{N} A] \mid v \in \Omega_{\alpha I}\right\rangle .
$$

We claim that $\mathcal{J}(X, \alpha I)$ can be computed using an analog of Howald's formula. Recall that the linear map $F: \mathbb{R}^{d} \rightarrow \mathbb{R}^{\mathscr{F}}$ induces ring homomorphisms

$$
\mathbb{k}[\mathbb{N} A] \stackrel{\sim}{\rightarrow} \mathbb{k}[F(\mathbb{N} A)] \rightarrow \mathbb{k}\left[\mathbb{N}^{\mathscr{F}}\right] .
$$

By abusing notation, denote

$$
F(I)=\mathbb{k}[F(\mathbb{N} A)] \cdot\left\langle x^{F(v)} \mid y^{v} \in I\right\rangle
$$

the ideal in $\mathbb{k}[F(\mathbb{N} A)]$ obtained from the semigroup isomorphism $F: \mathbb{N} A \stackrel{\sim}{\rightarrow} F(\mathbb{N} A)$. The monomial ideal in $\mathbb{k}\left[\mathbb{N}^{\mathscr{F}}\right]$ generated by $F(I)$ is denoted by

$$
J=\mathbb{k}\left[\mathbb{N}^{\mathscr{F}}\right] \cdot\langle F(I)\rangle .
$$

Let $e$ be the element in $\mathbb{R}^{\mathscr{F}}$ such that $e_{\sigma}=1$ for all $\sigma \in \mathscr{F}$. Notice that $e$ may not be in $F(\mathbb{N} A)$ and that $e \in F(\mathbb{N} A)$ if and only if $X$ is Gorenstein. Even if one extends to rational coefficients, the element $e$ may not be in $F(\mathbb{Q} \otimes \mathbb{N} A)=F\left(\mathbb{Q}^{d}\right)$. The condition $e \in F\left(\mathbb{Q}^{d}\right)$ holds exactly when $X$ is $\mathbb{Q}$-Gorenstein, namely $K_{X}$ is a $\mathbb{Q}$-Cartier divisor. This is the case when $X$ is $\mathbb{Q}$-factorial (or, equivalently, the semigroup $\mathbb{N} A$ is simplicial).

In the case where $e \in F\left(\mathbb{Q}^{d}\right)$, it is clear that $\Omega_{\alpha I}=\Omega_{F^{-1}(-e), \alpha I}$, so $w=F^{-1}(-e)$ corresponds to the $\Delta$ of De Fernex and Hacon. In fact, the divisor $\Delta_{F^{-1}(-e)}=0$ coincides with the canonical choice of boundary divisor for the pair $(X, \alpha I)$ in the $\mathbb{Q}$-Gorenstein case. Moreover, we have the following analog of Howald's formula for $\mathcal{J}(X, \alpha I)$.

Proposition 4.1. If $X$ is $\mathbb{Q}$-Gorenstein, then

$$
\mathcal{J}(X, \alpha I)=\left\langle y^{v} \in \mathbb{k}[\mathbb{N} A] \mid F(v)+e \in \operatorname{int}\left(\alpha P_{F(I)}\right)\right\rangle .
$$

Proof.

$$
\begin{aligned}
\mathcal{J}(X, \alpha I) & =\left\langle y^{v} \in \mathbb{k}[\mathbb{N} A] \mid v \in \Omega_{\alpha I}\right\rangle \\
& =\left\langle y^{v} \in \mathbb{k}[\mathbb{N} A] \mid v \in \Omega_{F^{-1}(-e), \alpha I}\right\rangle \\
& =\left\langle y^{v} \in \mathbb{k}[\mathbb{N} A] \mid F(v)+e \in \operatorname{int}\left(\alpha F\left(P_{I}\right)\right)\right\rangle \\
& =\left\langle y^{v} \in \mathbb{k}[\mathbb{N} A] \mid F(v)+e \in \operatorname{int}\left(\alpha P_{F(I)}\right)\right\rangle .
\end{aligned}
$$

Since $P_{I}=\operatorname{conv}\left[\bigcup_{v: y^{v} \in I}(v+C)\right]$ is the convex hull in $\mathbb{R}^{d}$ of the set $\bigcup_{y^{v} \in I}(v+C)$, we see that $F\left(P_{I}\right)=\operatorname{conv}\left[\bigcup_{v: y^{v} \in I}(F(v)+F(C))\right]$ is exactly the Newton polyhedron $P_{F(I)}$ in $F\left(\mathbb{R}^{d}\right)$ of the ideal $F(I)$ in the semigroup ring $\mathbb{k}[F(\mathbb{N} A)]$. The last expression in (4.5) is analogous to Howald's formula. 
Notations for Newton Polytopes. For a monomial ideal $I$ in $\mathbb{k}[\mathbb{N} A] \subset \mathbb{k}\left[y_{1}^{ \pm 1}, \ldots, y_{d}^{ \pm 1}\right]$, denote $P_{I}$ the Newton polyhedron of $I$, which by definition is the convex hull of $\bigcup_{v: y^{v} \in I}(v+\mathbb{N} A)$ in $\mathbb{R}^{d}$. The relative interior of $P_{I}$ is denoted by int $P_{I}$.

Recall from (4.3) that $F(I)$ denotes a monomial ideal in $\mathbb{k}[\mathbb{N} A]$. The Newton polyhedron of $F(I)$ is denoted by $P_{F(I)}$; this is the convex hull of $\left\{F(v) \mid y^{v} \in I\right\}$ in $F\left(\mathbb{R}^{d}\right)$. We have

$$
P_{F(I)}=\operatorname{conv}\left[\bigcup_{v: y^{v} \in I}(F(v)+F(\mathbb{N} A))\right] .
$$

Recall also from (4.4) that $J=\mathbb{k}\left[\mathbb{N}^{\mathscr{F}}\right] \cdot\langle F(I)\rangle$ denotes the monomial ideal in $\mathbb{k}\left[\mathbb{N}^{\mathscr{F}}\right]$ generated by $F(I)$. The Newton polyhedron of $J$ in $\mathbb{R}^{\mathscr{F}}$ is

$$
P_{J}=\operatorname{conv}\left[\bigcup_{v: y^{v} \in I}\left(F(v)+\mathbb{N}^{\mathscr{F}}\right)\right] .
$$

Note that if $y^{\beta_{1}}, \ldots, y^{\beta_{r}}$ is the set of minimal monomial generators of $I$, then $\left\{\beta_{1}, \ldots, \beta_{r}\right\}$ (respectively, $\left\{F\left(\beta_{1}\right), \ldots, F\left(\beta_{r}\right)\right\}$ ) is exactly the set of vertices of the Newton polyhedron $P_{I}$ (respectively, $P_{F(I)}$ or $\left.P_{J}\right)$.

For general $X$, we give a description of $\mathcal{J}(X, \alpha I)$ using the Newton polyhedron of the ideal $J=\mathbb{k}\left[\mathbb{N}^{\mathscr{F}}\right] \cdot\langle F(I)\rangle$. We first need to compare the relative interiors of $\alpha P_{F(I)}$ and $\alpha P_{J}$.

Lemma 4.2. For general $\mathbb{N} A$, the relative interiors of $P_{F(I)}$ and $P_{J}$ satisfy

$$
\operatorname{int}\left(\alpha P_{F(I)}\right)=F(C) \cap \operatorname{int}\left(\alpha P_{J}\right) \text {. }
$$

Proof. A point $u$ lies in $\operatorname{int}\left(P_{F(I)}\right)$ if and only if $u-v \in \operatorname{int}(F(C))$ for some $v$ on a bounded face of $P_{F(I)}$. Similarly, a point $u$ lies in $\operatorname{int}\left(P_{J}\right)$ if and only if $u-v \in \operatorname{int} \mathbb{R}_{\geq 0}^{\mathscr{F}}$ for some $v$ on a bounded face of $P_{J}$. Since $F(I)$ and $J$ have the same minimal monomial generators, the bounded faces of $P_{F(I)}$ and $P_{J}$ coincide. Therefore, it suffices to show that

$$
\operatorname{int}(F(C))=F(C) \cap \operatorname{int}\left(\mathbb{R}_{\geq 0}^{\mathscr{F}}\right) .
$$

But for $p \in C, F(p) \in \operatorname{int} F(C)$ if and only if $p$ is not contained in any facet $\sigma \in \mathscr{F}$, which means exactly $F_{\sigma}(p)>0$.

Proposition 4.3. The multiplier ideal

$$
\mathcal{J}(X, \alpha I)=\left\langle y^{v} \in \mathbb{k}[\mathbb{N} A] \mid F(v)+e \in \operatorname{int}\left(\alpha P_{J}\right)\right\rangle .
$$

Proof. If $X$ is $\mathbb{Q}$-Gorenstein, the statement follows from Proposition 4.1 and Lemma 4.2. In general, by (4.1) and (4.2) it suffices to show that

$$
F\left(\Omega_{\alpha I} \cap \mathbb{N} A\right)=\left(-e+\operatorname{int}\left(\alpha P_{J}\right)\right) \cap F(\mathbb{N} A) .
$$

For the containment $F\left(\Omega_{\alpha I} \cap \mathbb{N} A\right) \subseteq\left(-e+\operatorname{int}\left(\alpha P_{J}\right)\right) \cap F(\mathbb{N} A)$, it is enough to verify that

$$
F\left(w+\operatorname{int}\left(\alpha P_{I}\right)\right) \subseteq\left[-e+\operatorname{int}\left(\alpha P_{J}\right)\right]
$$


for any $w \in \mathbb{Q}^{d}$ satisfying $F_{\sigma}(w) \geq-1$ for all $\sigma \in \mathscr{F}$. Any such $w$ satisfies $F(w)+e \in \mathbb{R}_{\geq 0}^{\mathscr{F}}$, so by Lemma 4.2

$$
\begin{aligned}
F\left(w+\operatorname{int}\left(\alpha P_{I}\right)\right) & =\left[F(w)+\operatorname{int}\left(\alpha F\left(P_{I}\right)\right)\right] \\
& =\left[F(w)+\operatorname{int}\left(\alpha P_{F(I)}\right)\right] \\
& \subseteq\left[F(w)+\operatorname{int}\left(\alpha P_{J}\right)\right] \\
& =\left[-e+(F(w)+e)+\operatorname{int}\left(\alpha P_{J}\right)\right] \\
& \subseteq\left[-e+\mathbb{R}_{\geq 0}^{\mathscr{F}}+\operatorname{int}\left(\alpha P_{J}\right)\right] \\
& \subseteq\left[-e+\operatorname{int}\left(\alpha P_{J}\right)\right] .
\end{aligned}
$$

For the other containment $F\left(\Omega_{\alpha I} \cap \mathbb{N} A\right) \supseteq\left(-e+\operatorname{int}\left(\alpha P_{J}\right)\right) \cap F(\mathbb{N} A)$, let $v \in \mathbb{N} A$ be such that $F(v)+e \in \operatorname{int}\left(\alpha P_{J}\right)$. Then there exists $u$ lying on a bounded face of $\alpha P_{I}$ such that

$$
F(v)+e-F(u) \in \operatorname{int}\left(\mathbb{R}_{\geq 0}^{\mathscr{F}}\right)
$$

In particular, $F_{\sigma}(v-u)>-1$ for all $\sigma \in \mathscr{F}$. Take any $p \in \operatorname{int} C$ and $\epsilon>0$ small enough so that

$$
w:=v-u-\epsilon p \in \mathbb{Q}^{d} \text { and } F_{\sigma}(v-u-\epsilon p)>-1 .
$$

Then $F(v)-F(u)-F(w)=F(\epsilon p) \in \operatorname{int} C$ where $F(u)$ lies on a bounded face of $\alpha P_{F(I)}$. Therefore, we have $F(v)-F(w) \in \operatorname{int}\left(\alpha P_{F(I)}\right)$, and hence $F(v) \in F\left(\Omega_{w, \alpha I} \cap \mathbb{N} A\right) \subseteq F\left(\Omega_{\alpha I} \cap\right.$ $\mathbb{N} A$ ) as desired.

4.2. Roots-Jumping coefficients correspondence on toric varieties. Now, we combine the previous observations to establish the following theorem.

Theorem 4.4. Let $\mathbb{N}$ A be a normal semigroup, $X=\operatorname{Spec}(\mathbb{K}[\mathbb{N} A])$ its associated affine toric variety and let $I$ be a monomial ideal on $X$. Suppose $\alpha_{I}$ is the smallest root of $b_{I}(-s)$ where $b_{I}(s)$ is the Bernstein-Sato polynomial of $I$ in $\mathbb{k}[\mathbb{N} A]$. Then any jumping coefficients of the pair $(X, I)$ in $\left[\alpha_{I}, \alpha_{I}+1\right)$ are roots of $b_{I}(-s)$. Moreover, the number $\alpha_{I}$ is the smallest jumping coefficient (i.e. the log-canonical threshold) of $(X, I)$.

Proof. By Proposition 3.2, the Berstein-Sato polynomial $b_{I}(s)$ of $I$ coincides with the BernsteinSato polynomial of the monomial ideal $J=\mathbb{k}\left[\mathbb{N}^{\mathscr{F}}\right] \cdot\langle F(I)\rangle$. Thus by [BMS06a, Theorem 2], the jumping coefficients of the pair $\left(\mathbb{A}^{\mathscr{F}}, J\right)$ in $\left[\alpha_{I}, \alpha_{I}+1\right)$ are roots of $b_{I}(-s)$. By Howald's formula, a number $\alpha$ is a jumping coefficient of $\left(\mathbb{A}^{\mathscr{F}}, J\right)$ exactly when the boundary of $\left(-e+\alpha P_{J}\right)$ contains a lattice point in $\mathbb{N}^{\mathscr{F}}$. Also, according to Proposition 4.3 a number $\alpha$ is a jumping coefficient of $(X, I)$ exactly when the boundary of $\left(-e+\alpha P_{J}\right)$ contains a lattice point in $F(\mathbb{N} A)$. Therefore, the jumping coefficients of $(X, I)$ are jumping coefficients of $\left(\mathbb{A}^{\mathscr{F}}, J\right)$, and the first statement of this theorem follows.

To prove $\alpha_{I}$ is the log-canonical threshold of $(X, I)$, it suffices to show that the boundary of $\left(-e+\alpha_{I} P_{J}\right)$ intersects $F(\mathbb{N} A)$. Since $\alpha_{I}$ is the log-canonical threshold of $\left(\mathbb{A}^{\mathscr{F}}, J\right)$, we have

$$
1 \in \mathbb{k}\left[\mathbb{N}^{\mathscr{F}}\right]=\mathcal{J}\left(\mathbb{A}^{\mathscr{F}}, \alpha_{I} J\right) \text {, and hence } F(0)=0 \in\left(-e+\alpha_{I} P_{J}\right) \cap F(\mathbb{N} A) \text {. }
$$

Example 4.5. Let $A=\left(\begin{array}{llll}1 & 1 & 1 & 1 \\ 0 & 1 & 2 & 3\end{array}\right)$ and $I=\left\langle y_{1} y_{2}, y_{1} y_{2}^{2}\right\rangle$ a monomial ideal in $\mathbb{k}[\mathbb{N} A]$. In this case, the semigroup ring $\mathbb{k}[\mathbb{N} A]$ is simplicial and hence $\mathbb{Q}$-Gorenstein. The linear mapping 
$F: \mathbb{R}^{2} \rightarrow \mathbb{R}^{2}$ is represented by the matrix $\left(\begin{array}{rr}3 & -1 \\ 0 & 1\end{array}\right)$. The subsemigroup $\mathbb{k}[F(\mathbb{N} A)]$ of $\mathbb{k}\left[x_{1}, x_{2}\right]$ is generated by $x_{1}^{3}, x_{1}^{2} x_{2}, x_{1} x_{2}^{2}, x_{2}^{3}$ and the monomial ideal $J=\mathbb{k}\left[x_{1}, x_{2}\right]\left\langle x_{1}^{2} x_{2}, x_{1} x_{2}^{2}\right\rangle$. Using the method of Budur, Mustaţă and Saito as discussed in subsection 2.2, one can compute the BernsteinSato polynomial $b_{J}(s)=(s+1)^{2}(3 s+2)(3 s+4)$. (We point out that $b$-function algorithms have been developed and implemented, see [BL10].) Moreover, by Proposition 3.2 we have $b_{I}(s)=$ $(s+1)^{2}(3 s+2)(3 s+4)$ as well. On the other hand, using Howald's formula, one finds that $\frac{2}{3}, 1, \frac{4}{3}$ are jumping coefficients of $\left(\mathbb{A}^{2}, J\right)$, but only $\frac{2}{3}, 1$ are jumping coefficients of $(X, I)$ according to Proposition 4.3 .

\section{ACKNOWLEDGEMENTS}

The first author thanks Texas A\&M University for the hospitality he enjoyed during his visit in January 2016, when this work was initiated.

\section{REFERENCES}

[Ber72] I. N. Bernšteřn, Analytic continuation of generalized functions with respect to a parameter, Funkcional. Anal. i Priložen. 6 (1972), no. 4, 26-40. MR 0320735 (47 \#9269) 1

[BGG72] I. N. Bernšteřn, I. M. Gel'fand, and S. I. Gel'fand, Differential operators on a cubic cone, Uspehi Mat. Nauk 27 (1972), no. 1(163), 185-190. MR 0385159 (52 \#6024) 5]

[BL10] Christine Berkesch and Anton Leykin, Algorithms for Bernstein-Sato polynomials and multiplier ideals, ISSAC 2010-Proceedings of the 2010 International Symposium on Symbolic and Algebraic Computation, ACM, New York, 2010, pp. 99-106. MR 2920542 13

[Bli04] Manuel Blickle, Multiplier ideals and modules on toric varieties, Math. Z. 248 (2004), no. 1, 113-121. MR 2092724 (2006a:14082) 3, 9

[BMS06a] Nero Budur, Mircea Mustaţă, and Morihiko Saito, Bernstein-Sato polynomials of arbitrary varieties, Compos. Math. 142 (2006), no. 3, 779-797. MR 2231202 (2007c:32036) 1, 2, 3, 4, 6, 12

[BMS06b] _ Combinatorial description of the roots of the Bernstein-Sato polynomials for monomial ideals, Comm. Algebra 34 (2006), no. 11, 4103-4117. MR 2267574 (2007h:32041) 2 , 6

[dFH09] Tommaso de Fernex and Christopher D. Hacon, Singularities on normal varieties, Compos. Math. 145 (2009), no. 2, 393-414. MR 2501423 (2010c:14013)1, 9

[ELSV04] Lawrence Ein, Robert Lazarsfeld, Karen E. Smith, and Dror Varolin, Jumping coefficients of multiplier ideals, Duke Math. J. 123 (2004), no. 3, 469-506. MR 2068967 (2005k:14004)1

[Gro67] A. Grothendieck, Éléments de géométrie algébrique. IV. Étude locale des schémas et des morphismes de schémas IV, Inst. Hautes Études Sci. Publ. Math. (1967), no. 32, 361. MR 02388604

[How01] J. A. Howald, Multiplier ideals of monomial ideals, Trans. Amer. Math. Soc. 353 (2001), no. 7, 2665-2671 (electronic). MR 18284663, 9

[Igu00] Jun-ichi Igusa, An introduction to the theory of local zeta functions, AMS/IP Studies in Advanced Mathematics, vol. 14, American Mathematical Society, Providence, RI; International Press, Cambridge, MA, 2000. MR 17434671

[Jon94] A. G. Jones, Rings of differential operators on toric varieties, Proc. Edinburgh Math. Soc. (2) 37 (1994), no. 1, 143-160. MR 1258039 (95d:16030)2, 5

[Kas83] M. Kashiwara, Vanishing cycle sheaves and holonomic systems of differential equations, Algebraic geometry (Tokyo/Kyoto, 1982), Lecture Notes in Math., vol. 1016, Springer, Berlin, 1983, pp. 134-142. MR 726425 (85e:58137)1

[Ko197] János Kollár, Singularities of pairs, Algebraic geometry—Santa Cruz 1995, Proc. Sympos. Pure Math., vol. 62, Amer. Math. Soc., Providence, RI, 1997, pp. 221-287. MR 14925251

[Laz04] Robert Lazarsfeld, Positivity in algebraic geometry. II, Ergebnisse der Mathematik und ihrer Grenzgebiete. 3. Folge. A Series of Modern Surveys in Mathematics [Results in Mathematics and Related Areas. 3rd Series. A Series of Modern Surveys in Mathematics], vol. 49, Springer-Verlag, Berlin, 2004, Positivity for vector bundles, and multiplier ideals. MR 2095472 (2005k:14001b) 18 
[Lic89] B. Lichtin, Poles of $|f(z, w)|^{2 s}$ and roots of the b-function, Ark. Mat. 27 (1989), no. 2, 283-304. MR 1022282[1]

[Mal83] B. Malgrange, Polynômes de Bernstein-Sato et cohomologie évanescente, Analysis and topology on singular spaces, II, III (Luminy, 1981), Astérisque, vol. 101, Soc. Math. France, Paris, 1983, pp. 243-267. MR 737934 (86f:58148) 1

[Mus87] Ian M. Musson, Rings of differential operators on invariant rings of tori, Trans. Amer. Math. Soc. 303 (1987), no. 2, 805-827. MR 902799 (88m:32019)2]5

[SS72] Mikio Sato and Takuro Shintani, On zeta functions associated with prehomogeneous vector spaces, Proc. Nat. Acad. Sci. U.S.A. 69 (1972), 1081-1082. MR 0296079 (45 \#5140) 1

[ST01] Mutsumi Saito and William N. Traves, Differential algebras on semigroup algebras, Symbolic computation: solving equations in algebra, geometry, and engineering (South Hadley, MA, 2000), Contemp. Math., vol. 286, Amer. Math. Soc., Providence, RI, 2001, pp. 207-226. MR 18742812, 5

[Yan83] Tamaki Yano, b-functions and exponents of hypersurface isolated singularities, Singularities, Part 2 (Arcata, Calif., 1981), Proc. Sympos. Pure Math., vol. 40, Amer. Math. Soc., Providence, RI, 1983, pp. 641652. MR 7132871

Mathematics Department, Cheng Kung University, Tainan City 70101, Taiwan

E-mail address: jhsiao@mail.ncku.edu.tw

Mathematics Department, Texas A\&M University, College Station, TX 77843

E-mail address: laura@math.tamu.edu 